\title{
Mechanisms and evolution of oxidative sulfur metabolism in green sulfur bacteria
}

\author{
Lea H. Gregersen ${ }^{1+}$, Donald A. Bryant ${ }^{2}$ and Niels-Ulrik Frigaard ${ }^{1 *}$ \\ Department of Biology, University of Copenhagen, Helsinger, Denmark \\ 2 Department of Biochemistry and Molecular Biology, The Pennsylvania State University, University Park, PA, USA
}

Edited by:

Martin G. Klotz, University of Louisville, USA

\section{Reviewed by:}

Ulrike Kappler, University of

Queensland, Australia

Jan Kuever, Bremen Institute for

Materials Testing, Germany

${ }^{*}$ Correspondence:

Niels-Ulrik Frigaard, Section for Marine

Biology, Department of Biology,

University of Copenhagen,

Strandpromenaden 5, DK-3000

Helsingør, Denmark.

e-mail:nuf@bio.ku.dk

tPresent address:

Lea H. Gregersen, Max-DelbrückCentrum für Molekulare Medizin,

Berlin-Buch, Robert-Rössle-Strasse 10, D-13092 Berlin, Germany
Green sulfur bacteria (GSB) constitute a closely related group of photoautotrophic and thiotrophic bacteria with limited phenotypic variation. They typically oxidize sulfide and thiosulfate to sulfate with sulfur globules as an intermediate. Based on genome sequence information from 15 strains, the distribution and phylogeny of enzymes involved in their oxidative sulfur metabolism was investigated. At least one homolog of sulfide:quinone oxidoreductase (SOR) is present in all strains. In all sulfur-oxidizing GSB strains except the earliest diverging Chloroherpeton thalassium, the sulfide oxidation product is further oxidized to sulfite by the dissimilatory sulfite reductase (DSR) system. This system consists of components horizontally acquired partly from sulfide-oxidizing and partly from sulfate-reducing bacteria. Depending on the strain, the sulfite is probably oxidized to sulfate by one of two different mechanisms that have different evolutionary origins: adenosine-5'-phosphosulfate reductase or polysulfide reductase-like complex 3. Thiosulfate utilization by the SOX system in GSB has apparently been acquired horizontally from Proteobacteria. SoxCD does not occur in GSB, and its function in sulfate formation in other bacteria has been replaced by the DSR system in GSB. Sequence analyses suggested that the conserved SOXJXYZAKBW gene cluster was horizontally acquired by Chlorobium phaeovibrioides DSM 265 from the Chlorobaculum lineage and that this acquisition was mediated by a mobile genetic element. Thus, the last common ancestor of currently known GSB was probably photoautotrophic, hydrogenotrophic, and contained SQR but not DSR or SOX. In addition, the predominance of the Chlorobium-Chlorobaculum-Prosthecochloris lineage among cultured GSB could be due to the horizontally acquired DSR and SOX systems. Finally, based upon structural, biochemical, and phylogenetic analyses, a uniform nomenclature is suggested for sqrgenes in prokaryotes.

Keywords: horizontal gene transfer, metabolism evolution, green sulfur bacteria, purple sulfur bacteria, sulfide:quinone oxidoreductase, flavocytochrome $c$, SOX enzyme system, dissimilatory sulfite reductase

\section{INTRODUCTION}

Green sulfur bacteria (GSB; order Chlorobiales) are strictly anaerobic, photosynthetic bacteria that predominantly oxidize reduced sulfur compounds for photoautotrophic growth (Overmann, 2000, 2008; Garrity and Holt, 2001). They occur in anoxic, aquatic environments where light and sulfide coincide. They utilize predominantly sulfide, thiosulfate, biogenic and abiogenic sulfur globules, and $\mathrm{H}_{2}$, as electron donors to support photoautotrophic growth (Brune, 1989; Frigaard and Dahl, 2009). One characterized GSB, Chlorobium ferrooxidans, utilizes $\mathrm{Fe}^{2+}$ as electron donor and does not grow on reduced sulfur compounds (Heising et al., 1999).

Based on isolated strains that have been characterized, GSB form a phylogenetically and physiologically coherent group (Imhoff, 2003, 2008). Following a recent proposal to reorganize the GSB nomenclature, the GSB currently comprise only four genera: Chlorobium (Chl.), Chlorobaculum (Cba.), Prosthecochloris (Ptc.), and Chloroherpeton (Chp.; Imhoff, 2003). The genus Pelodictyon (Pld.), previously used for some GSB, has been abandoned. Available SSU rRNA sequences from cultured GSB exhibit relatively little variation: there is at least 92\% sequence identity among Chlorobium, Chlorobaculum, and Prosthecochloris and at least $88 \%$ sequence identity when Chloroherpeton is included. GSB constitute the only cultured members of the order Chlorobiales, and the phylum Chlorobi was proposed based on GSB (Garrity and Holt, 2001; Imhoff, 2008). Until Ignavibacterium album was isolated, the closest cultured relatives of the GSB were members of the Bacteroidetes (Iino et al., 2010). I. album is a non-phototrophic relative of GSB (about $82-83 \%$ SSU rRNA sequence identity with GSB; Iino et al., 2010), whose genome has recently been sequenced to reveal more about the physiology and evolution of this organism. Metagenomic sequence data derived from microbial mats of hot springs in Yellowstone National Park suggest that these mats harbor other uncharacterized relatives of GSB (Klatt et al., 2011; Liu et al., 2011). Preliminary analyses suggest that these organisms are photoheterotrophs that are physiologically distinct from well-studied Chlorobiales (Bryant et al., 2011).

The genome sequences of 15 strains of GSB are currently available (Table 1). Twelve of these genome sequences are publicly available ${ }^{1}$, one other has been completed (Ptc. bathyomarinum Ty

'http://ncbi.nlm.nih.gov 
Vent), and two are at the draft stage (Ptc. phaeum CIB 2401 and Cba. limnaeum DSM 1677; Bryant et al., 2011). Three of these strains were previously referred to as members of the Chlorobium (Prosthecochloris sp. BS1, Ptc. phaeum CIB 2401, and Ptc. bathyomarinum Ty Vent). However, phylogenetic sequence analyses of SSU rRNA and protein-encoding genes of these strains clearly show that they are more closely related to members of the genus Prosthecochloris than to members of the genera Chlorobium and Chlorobaculum (see below).

A defining feature of GSB as a group is their ability to oxidize inorganic sulfur compounds for photoautotrophic growth. The metabolic reactions involved in this dissimilatory sulfur metabolism are complex and have not yet been described in detail (Figure 1; Frigaard and Dahl, 2009). However, genome sequence information has provided significant insights into this metabolism, including which genes are likely to be involved and how they have evolved (Eisen et al., 2002; Chan et al., 2008; Frigaard and Bryant, 2008; Sakurai et al., 2010). Several enzymes from GSB have been identified using this information that have, or may have, a role in thiotrophic growth (e.g., Hanson and Tabita, 2001; Ogawa et al., 2008; Azai et al.,
2009; Chan et al., 2009; Holkenbrink et al., 2011). Using genome sequence analysis, we have investigated the distribution of putative sulfur metabolism genes in all currently available genomes of GSB.

\section{MATERIALS AND METHODS SEQUENCE DATA AND ANALYSIS}

Genome sequencing and annotation of GSB strains Cba. limnaeum DSM 1677, Ptc. bathyomarinum Ty Vent, and Ptc. phaeum CIB 2401 are currently being performed in our laboratories. Genome sequence information of PSB strains Ectothiorhodospira (Ect.) haloalkaliphila ATCC 51935, Marichromatium (Mch.) purpuratum DSM 1591, Thiococcus (Tco.) pfennigii 4520, Thiocystis (Tcs.) violascens DSM 198, Thiodictyon sp. Cad16, Thioflavicoccus (Tfc.) mobilis 8321, and Thiorhodospira (Trs.) sibirica ATCC 700588 was obtained from Joint Genome Institute, U.S. Department of Energy ${ }^{2}$. Genome sequence information from other bacteria was retrieved from GenBank ${ }^{3}$. Sequence analysis was performed primarily using tools available at

${ }^{2} \mathrm{http}: / / w w w . j g i . d o e . g o v /$

${ }^{3}$ http://www.ncbi.nlm.nih.gov/

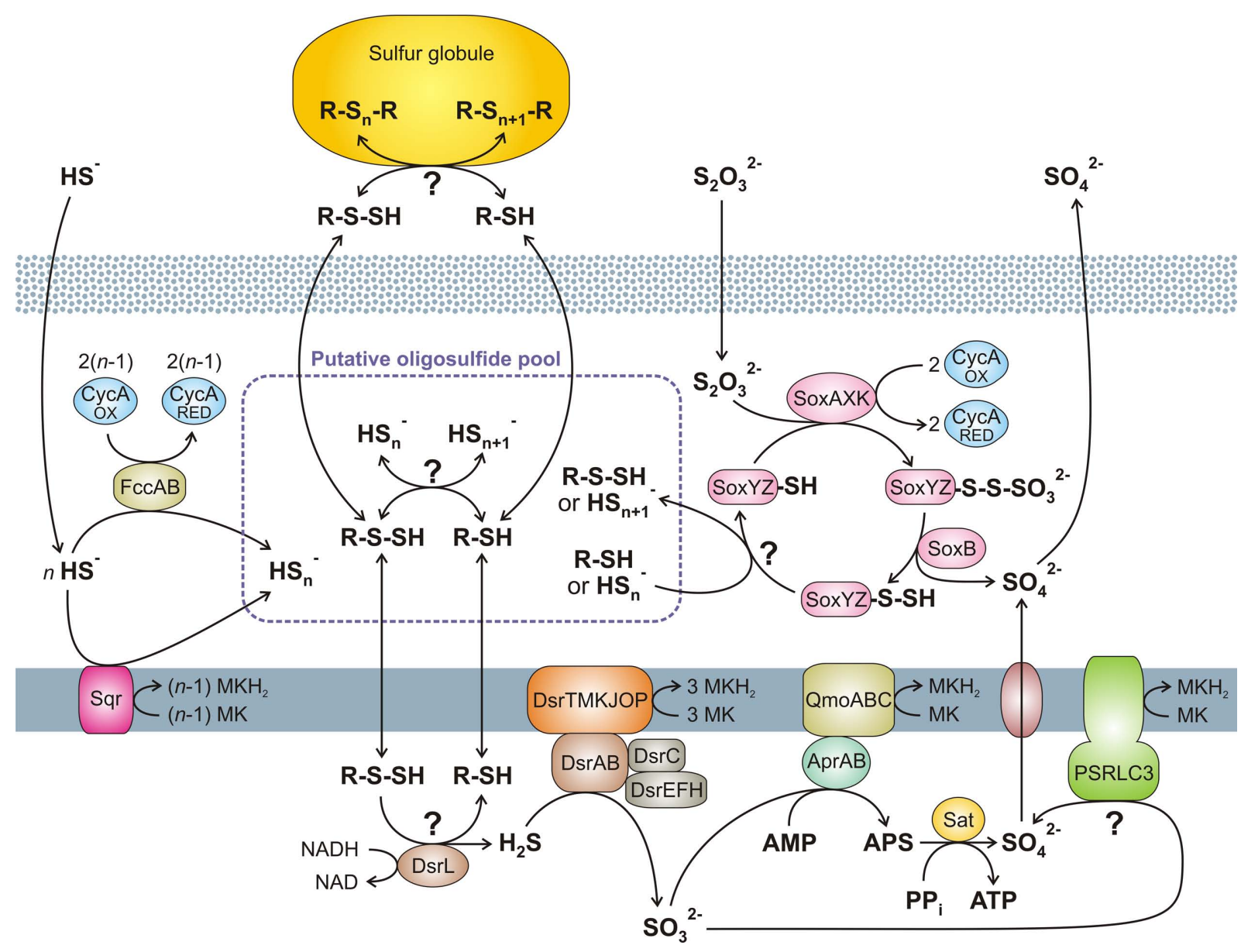

FIGURE 1 | Overview of the putative pathways of oxidative sulfur metabolism in GSB. All oxidative enzyme systems shown in the periplasm (SOX, SQR, and FCC systems) as well as extracellular sulfur globules contribute to a putative pool of oligosulfides ( $\mathrm{HS}_{\mathrm{n}}^{-}$and possibly organic R-S $-\mathrm{H}$ ). Complete oxidation of this pool to sulfate is dependent on the DSR system. No strain of GSB have all the reactions shown here. See text for further details. (Based on Eisen et al., 2002; Frigaard and Bryant, 2008; Frigaard and Dahl, 2009; Sakurai et al., 2010; Holkenbrink et al., 2011.) MK, menaquinone. 
the National Center for Biotechnology Information ${ }^{4}$ (NCBI) and at Integrated Microbial Genomes ${ }^{5}$ (IMG). Analyses were performed locally primarily using the following software: MEGA 4 (sequence alignment and phylogenetics ${ }^{6}$; Tamura et al., 2007), Artemis 12.0 (sequence viewing and manipulation; Wellcome Trust Sanger Institute, Hinxton, $\mathrm{UK}^{7}$; Carver et al., 2008), FGENESB (sequence annotation; Softberry, Mount Kisco, NY, USA ${ }^{8}$ ), and ActivePerl 5.10 (ActiveState Software, Vancouver, BC, Canada ${ }^{9}$ ). Prediction of signal peptides was performed using SignalP ${ }^{10}$; Emanuelsson et al., 2007) and PRED-TAT ${ }^{11}$; Bagos et al., 2010). Promoter regions were predicted using FGENESB and $\mathrm{BPROM}^{12}$ ) and manually edited. The sequence logo of the sox promoter region was generated using WebLogo ${ }^{13}$ version 2.8.2 (Crooks et al., 2004).

\section{RESULTS}

\section{PHYLOGENY OF RIBOSOMAL RNA AND CORE METABOLIC FUNCTIONS}

Analyses of the SSU rRNA gene and several cellular core proteins in the GSB strains with sequenced genomes revealed an overall congruent phylogeny (Figures 2A,B), which was in agreement with previous analyses performed on a smaller number of strains (Bryant et al., 2011). These analyses strongly supported the recent reclassification of GSB into four genera (Imhoff, 2003): Chlorobium, Chlorobaculum, Prosthecochloris, and Chloroherpeton. Chloroherpeton was consistently the earliest diverging genus within the Chlorobiales, Prosthecochloris was a sister clade to the Chlorobium/Chlorobaculum lineage, and Chlorobium and Chlorobaculum were the most closely related genera.

Phylogenetic analyses were made on selected enzymes functioning in several key metabolic processes in GSB. For example, analysis of PetB, the cytochrome $b$ subunit of the Rieske-cytochrome $b$ electron-transfer complex (PetB/CT0303; the locus tags for proteins from $\mathrm{Cba}$. tepidum are provided to facilitate identification), showed that this enzyme is present in all strains and that its phylogeny is congruent, at least at the genus level (Figure 2C), with that of the SSU rRNA (Figure 2A) and with a phylogeny based on the concatenation of several proteins representing various house-keeping functions of these organisms (Figure 2B). Therefore, PetB was most likely present in the last common ancestor of the investigated GSB, and the petB gene probably has not undergone horizontal transfer (at least not between members of different genera). Identical analyses and conclusions were made for the following enzymes, which are present in the genomes of all sequenced strains of GSB: ATP-citrate lyase subunit 2 (AclA/CT1088), type I NADH dehydrogenase (concatenated NuoDHLMN/CT0769,CT0770,CT0774-CT0776), ferredoxin-NAD $(\mathrm{P})^{+}$reductase (FNR/CT1512), RuBisCO-like

\footnotetext{
${ }^{4}$ http://www.ncbi.nlm.nih.gov/

${ }^{5}$ http://img.jgi.doe.gov/

${ }^{6}$ http://www.megasoftware.net/

${ }^{7}$ http://www.sanger.ac.uk/

${ }^{8} \mathrm{http}: / /$ linuxl.softberry.com/

${ }^{9}$ http://www.activestate.com/

${ }^{10}$ http://www.cbs.dtu.dk/services/SignalP/

${ }^{11}$ http://www.compgen.org/tools/PRED-TAT/

${ }^{12} \mathrm{http}$ ///linux1.softberry.com

${ }^{13}$ http://weblogo.berkeley.edu/
}

protein (RLP/CT1772), and nitrogen fixation (concatenated NifAHDKENB/CT1529,CT1533,CT1536-CT1540; data not shown). (Chp. thalassium ATCC 35110 has two nif gene sets: one encoding [Mo-Fe]-nitrogenase and one encoding Fe-only nitrogenase; the latter was probably horizontally acquired.) Similar observations have previously been made with the FennaMatthews-Olson antenna protein (FMO/CT1499; Alexander et al., 2002; Imhoff, 2003) and magnesium chelatase large subunits (BchH/CT1957, BchS/CT1955, BchT/CT1295; Gomez Maqueo Chew et al., 2009). The same is true for the phylogeny of the HupSL [Ni-Fe]-hydrogenase (HupL/CT0777; data not shown), except that the hupSL genes have been lost in a few strains (Table 1).

Type I NADH dehydrogenase is the enzyme that normally couples oxidation of $\mathrm{NADH}$ to the reduction of menaquinone (or another isoprenoid quinone, depending on the organism) and thereby produces a transmembrane proton gradient (Yagi and Matsuno-Yagi, 2003; Sazanov and Hinchliffe, 2006; Efremov et al., 2010). The bacterial enzyme normally has 14 subunits, NuoABCDEFGHIJKLMN. With the exception of Chp. thalassium, all GSB have lost three subunits, NuoE, NuoF, and NuoG (Frigaard et al., 2003), which function in binding and oxidation of $\mathrm{NADH}$ (Yagi and Matsuno-Yagi, 2003; Sazanov and Hinchliffe, 2006; Efremov et al., 2010). Phylogenetic analysis suggests that the loss occurred after divergence of Chloroherpeton spp., because both Chp. thalassium and the earlier diverging I. album have all 14 subunits (data not shown). This loss could have important consequences for the electron-transfer function of this enzyme and is likely to represent a major physiological difference between Chloroherpeton spp. and other GSB, which could impact the oxidative sulfur metabolism.

In summary, phylogenetic analyses strongly support the division of the GSB into only four genera: Chlorobium, Chlorobaculum, Prosthecochloris, and Chloroherpeton. In addition, the observations support the notion that the last common ancestor of extant, cultured GSB was an anaerobic bacteriochlorophyll-containing photoautotroph capable of $\mathrm{N}_{2}$ fixation, $\mathrm{H}_{2}$ uptake, and $\mathrm{CO}_{2}$ fixation by the reverse TCA cycle. Finally, these metabolic functions do not appear to have been exchanged by horizontal gene transfer in members of this clade. In contrast, many sulfur metabolism enzymes were apparently not present in the last common ancestor of extant GSB, and the genes encoding this metabolism have undergone extensive horizontal exchange with other bacterial phyla (see below).

\section{SULFIDE:OUINONE OXIDOREDUCTASES}

All characterized sulfide:quinone oxidoreductases (SQR) are single-subunit flavoproteins that are associated with the cytoplasmic membrane (Shahak and Hauska, 2008; Frigaard and Dahl, 2009). Based on the protein structure, a recently proposed classification identified six distinct types (numbered I through VI) of SQR homologs (Marcia et al., 2010). We propose a coherent genetic nomenclature that distinguishes among these SQR homologs in GSB and other organisms (Table 1; Figure 3). This nomenclature is necessary to identify clearly the multiple types of sqr genes often found in the same strain (Demerec et al., 1966).

We propose the gene designation sqrA for the type ISQR orthologs, which are typically found in Cyanobacteria, Proteobacteria, and Aquificaceae. This category includes the functionally wellcharacterized SQRs in Oscillatoria limnetica (AAF72962; Bronstein 

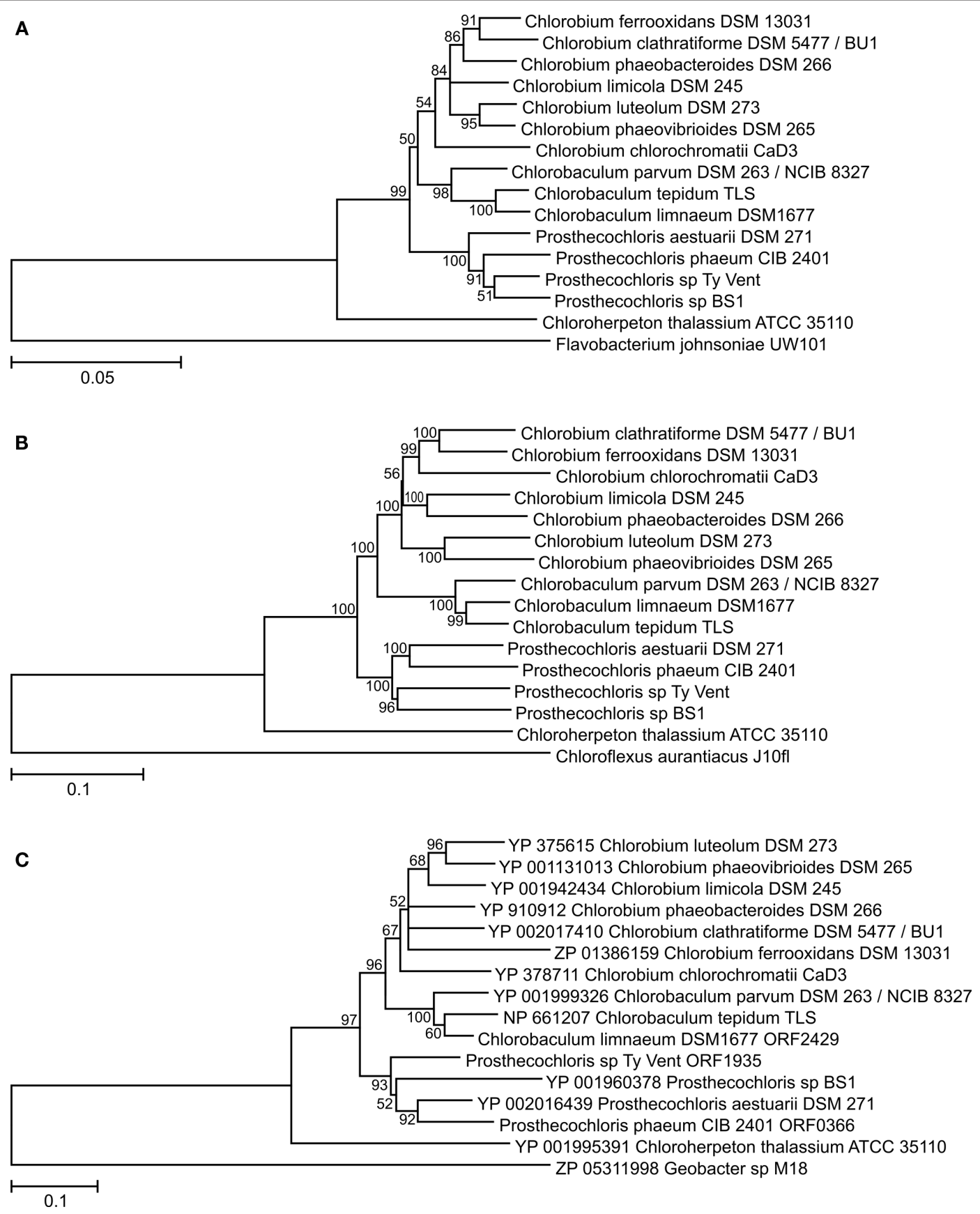

FIGURE 2 | Phylogenetic trees of ribosomal RNA and core metabolism enzymes of GSB. (A) SSU rRNA gene; rooted with Flavobacterium johnsoniae. (B) Concatenation of the following house-keeping proteins (with the protein name in Cba. tepidumTLS in parenthesis): RNA polymerase subunit beta (RpoB/CT0155), alanyl-tRNA synthetase (AlaS/CT0166), translation initiation factor IF-2 (InfB/CT0241), DNA gyrase subunit B (GyrB/CT2263), molecular chaperone DnaK (CT0643), $F_{0} F_{1}$ ATP synthase subunit $\alpha$ (AtpA/CT2033), preprotein translocase subunit SecY (CT2169), cell division protein FtsZ (CT0030), phospho-N-acetylmuramoyl-pentapeptide-transferase (MraY)
CT0037), recombinase A (RecA/CT1930); rooted with Chloroflexus aurantiacus J-10-fl. (C) Cytochrome b subunit of the Rieske-cytochrome b electron-transfer complex (PetB/CT0303); rooted with Geobacter sp. M18. All trees are made using the neighbor-joining method and the Jukes-Cantor (nucleotides) or Jones-Taylor-Thornton (amino acids) substitution model. All positions containing alignment gaps and missing data were eliminated only in pairwise sequence comparisons. Bootstrap support of branches in percentage based on 1000 replications are shown; branches with bootstrap values less than $50 \%$ were collapsed. et al., 2000), Rhodobacter capsulatus (CAA66112; Schütz et al., 1999), and Aquifex aeolicus (NP_214500; Nübel et al., 2000; Marcia et al., 2009). No GSB strain has a type I SQR (i.e., SqrA).
We propose the gene designation $s q r B$ for the type II SQR orthologs, which include SQR homologs identified in eukaryotes such as the functionally characterized SQR in Schizosaccharomyces 


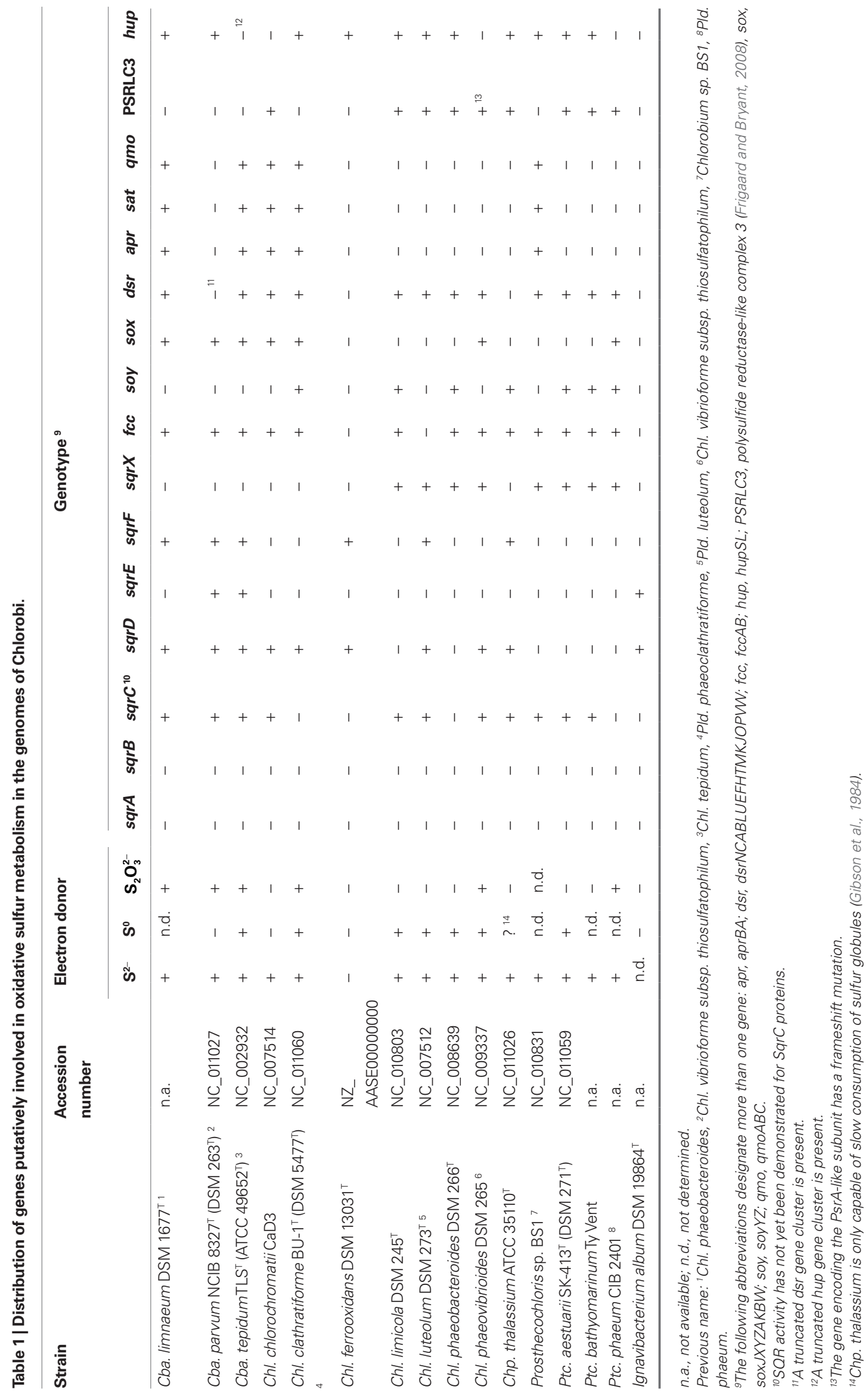




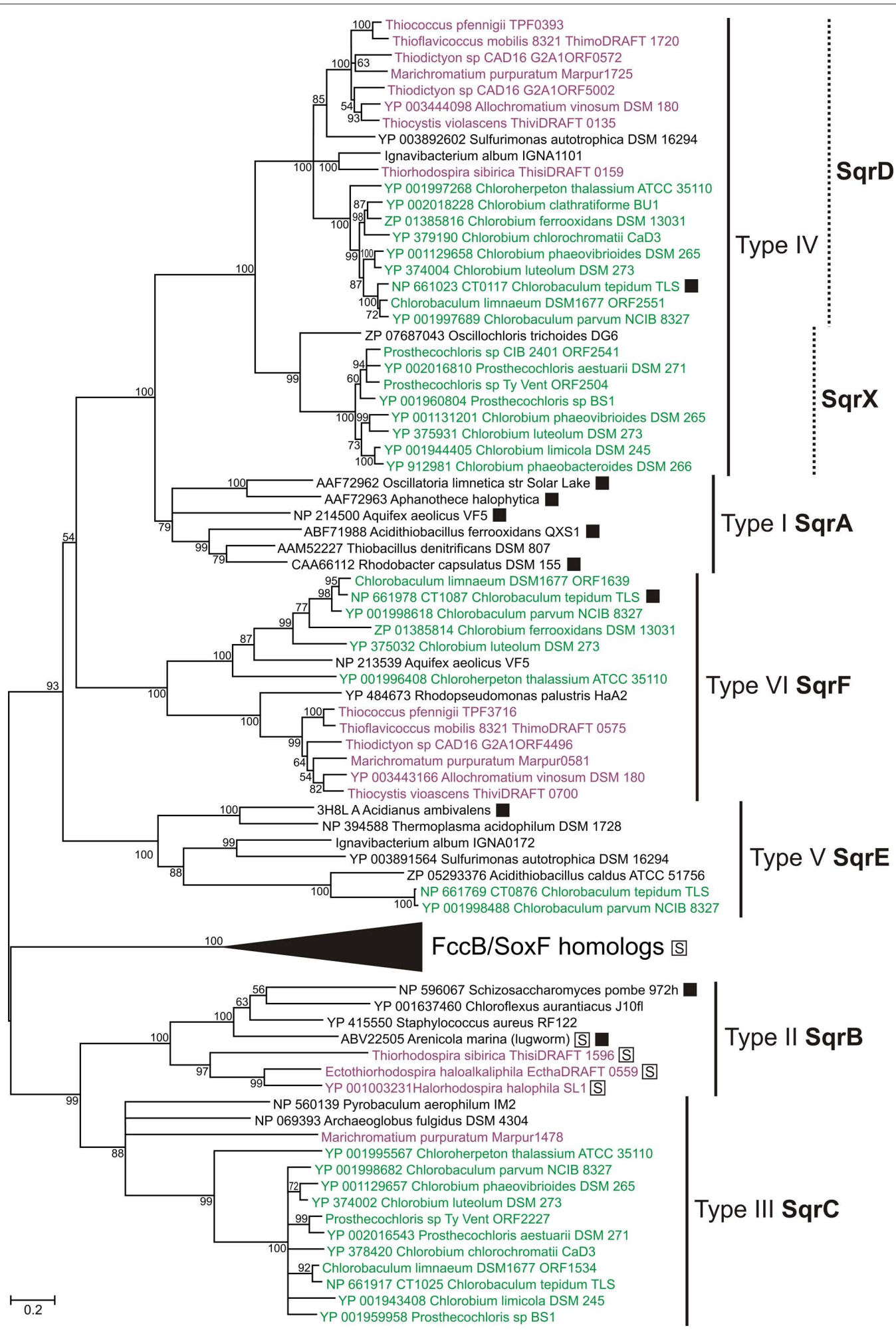

FIGURE 3 | Phylogenetic tree of sulfide:quinone oxidoreductase (SOR) homologs. Tree construction details were as in Figure 2, except that the tree is unrooted. The clade of FccB/SoxF homologs is expanded in Figure 4A. SQR activity has been demonstrated for proteins marked with a filled box. Proteins marked with a boxed "S" had a signal peptide. All proteins in the FccB/SoxF homologs clade (see Figure $4 A$ ) and proteins from PSB in the
SqrB clade had a signal peptide for the twin-arginine translocon (data not shown). The only other sequence in the tree that encoded a signal peptide of any predictable type (Emanuelsson et al., 2007) was the eukaryotic SQR type II from the lugworm Arenicola marina (data not shown). Sequences from GSB are shown in green; sequences from PSB are shown in purple. See text for details. 
pombe (NP_596067; Van de Weghe and Ow, 1999) and SQR homologs in the bacteria Staphylococcus aureus (YP_415550) and Chloroflexus aurantiacus (YP_001637460). No GSB strain has a type II SQR (i.e., SqrB).

We propose that the gene designation $s q r C$ should be reserved for the group of SQR homologs that were assigned as type III by Marcia et al. (2010). These proteins are the only SQR homologs for which no representative has yet been shown to exhibit SQR activity. The sulfide oxidation activity of a Cba. tepidum mutant lacking a type III protein (CT1025/NP_661917) was not significantly lower than that of the wild type (Holkenbrink, 2010).

We propose the gene designation sqrD for type IV SQR, which includes the SQR that is responsible for most of the SQR activity in Cba. tepidum(CT0117/NP_661023; Chan et al., 2009; Holkenbrink et al., 2011). Orthologs of SqrD are also present in Proteobacteria and Actinobacteria. Phylogenetic analyses show that structure class IV splits into two paralogous clades, and we propose the designation $s q r X$ for the second of these (see Figure 3). Examples of the SqrX family are present in about half of the sequenced GSB strains (e.g., YP_001944405 in Chlorobium limicola DSM 245) and thiotrophic Aquificaceae.

We propose the gene designation sqrE for type V SQR, which designates homologs of the functionally characterized SQR in the archaeon Acidianus ambivalens (3H8L; Brito et al., 2009). Cba. tepidum has an SqrE homolog (CT0876/NP_661769), although no SQR activity has been demonstrated for this protein (Chan et al., 2009).

We propose the gene designation sqrF for type VI SQR, which includes homologs of an SQR that has been functionally characterized in Cba.tepidum (CT1087/NP_661978; Chan et al., 2009).SqrF is important for growth of Cba. tepidum at high sulfide concentrations $(\geq 4 \mathrm{mM})$. Orthologs of SqrF are also present in thiotrophic Proteobacteria and Aquificaceae.

In summary, no GSB strain characterized to date has SQR enzymes belonging to structure classes I (SqrA) or II (SqrB), and enzymes of structure class $\mathrm{V}$ (SqrE) were only found in two Chlorobaculum spp. (Table 1). All GSB have at least one SQR homolog of structure class IV (SqrD or SqrX) or structure class VI (SqrF; Table 1), which generally appear to be characteristic for thiotrophic bacteria and archaea (Figure 3; Marcia et al., 2010). Because the types of SQR (III, IV, and VI) in Chp. thalassium are the earliest diverging SQR sequences in GSB in their respective clades (Figure 3), these types of SQR may have been present in the last common ancestor of the GSB investigated here.

Similar to most GSB, all PSB of the Chromatiaceae investigated (Alc. vinosum DSM 180, Mch. purpuratum DSM 1591, Tco. pfennigii 4520, Tcs. violascens DSM 198, Tfc. mobilis 8321, and Thiodictyon sp. Cad16) have both SqrD and SqrF (Figure 3). In contrast and unlike other PSB and GSB, all PSB of the Ectothiorhodospiraceae investigated (Ect. haloalkaliphila ATCC 51935, Hlr. halophila SL1, and Trs. sibirica ATCC 700588) have SqrB (Figure 3). The only exception to this pattern is Trs. sibirica, which contains both SqrB and SqrD (Figure 3). It is striking that the type of SQR in all of these PSB is clearly correlated with the location of the sulfur globules produced by the organisms: SqrD-containing strains (i.e., all Chromatiaceae) produce intracellular sulfur globules and SqrB-containing strains (i.e., all Ectothiorhodospiraceae) produce extracellular sulfur glob- ules. Trs. sibirica has both SqrB and SqrD, and this organism has been reported to produce both intracellular and extracellular sulfur globules (Bryantseva et al., 1999). In addition, only PSB that deposit intracellular sulfur globules (Chromatiaceae and Trs. sibirica) have homologs of the sulfur globule proteins SgpA and SgpB, which are absent from other PSB and from all GSB (data not shown). Described bacterial SQR sequences in general do not contain an N-terminal signal peptide (Shahak and Hauska, 2008; Marcia et al., 2010). Nevertheless, SqrA from Rhodobacter capsulatus and Aquifex aeolicus, which do not have an N-terminal signal peptide, are associated with the cytoplasmic membrane with the sulfide-binding site in the periplasm (Schütz et al., 1999; Marcia et al., 2009). It has been suggested that these proteins are translocated by an unknown mechanism that depends on a motif at the C-terminus of the SqrA polypeptide (Schütz et al., 1999). Surprisingly, all SqrB sequences of the Ectothiorhodospiraceae investigated here (Ect. haloalkaliphila, Hlr. halophila, and Trs. sibirica; Figure 3 ) had a signal peptide for the twin-arginine translocation (TAT; see Materials and Methods), which indicates that these proteins are translocated across the cytoplasmic membrane using the TAT pathway for proteins that require cytoplasmic assembly of a cofactor and folding prior to translocation. This implies that the active site of SqrB proteins also most likely resides in the periplasmic space. In conclusion, different types of SQR polypeptides are apparently targeted by different mechanisms. Furthermore, at least based on the nine PSB genomes analyzed here, the location of the deposited sulfur globules in PSB can be predicted by the type of the SQR that is present (SqrB or SqrD). This interpretation does not extend to GSB, because all GSB have type IV SQR (SqrD/SqrX) and not SqrB, and GSB produce only extracellular sulfur globules. The difference(s) responsible for SQR targeting and sulfur deposition between the PSB and GSB systems is currently not understood.

With respect to other phototrophic bacteria, purple non-sulfur bacteria and Cyanobacteria typically have SqrA and members of the Chloroflexi typically have SqrB.

\section{FLAVOCYTOCHROME CAND SOXF HOMOLOGS}

Flavocytochrome $c$ in GSB and PSB consists of a large, sulfidebinding, flavoprotein subunit (FccB) and a small, $c$-type cytochrome subunit (FccA; Kusai and Yamanaka, 1973; Brune, 1989; Reinartz et al., 1998). Although the exact physiological function is still debated, the enzyme is located in the periplasm and appears to be involved in oxidative sulfur metabolism. In Alc. vinosum this complex is encoded by $f c c A B$ (Reinartz et al., 1998). The homologous genes in Paracoccus pantotrophus and other bacteria are denoted soxEF (Friedrich et al., 2008). Here, we denote the genes $f c c A B$ if they occur in phototrophic sulfur bacteria (i.e., GSB or PSB) and soxEF in all other organisms. Almost all GSB have $f c c A B$ genes encoding flavocytochrome $c$ (Table 1). The $f c c A B$ genes do not cluster with sox genes in GSB, as is the case in P. pantotrophus and many other bacteria that contain sox genes (Friedrich et al., 2008). GSB that contain soy $Y Z$ genes may be considered to be an exception to this observation (Table 1). The soy $Y Z$ genes encode a putative, periplasmic SoyYZ complex that is paralogous to the SoxYZ complex, and SoyYZ may be involved in sulfur chemistry that does not involve either the SoxAX or SoxB proteins (Frigaard 
and Bryant, 2008). When the soyYZ genes are present in a GSB strain, they always form the cluster soy $Y Z-f c c A B$, which strongly suggests that a functional link exists between FccAB and SoyYZ.

Some GSB encode an additional homolog of FccB/SoxF that we denote as SoxJ. Some authors have denoted this protein as SoxF (Ogawa et al., 2010) or SoxF2 (Verte et al., 2002). However, we prefer to denote this protein as SoxJ to distinguish it clearly from SoxF that is a subunit of the SoxEF/FccAB flavocytochrome $c$ (see previous paragraph). The soxJ gene in GSB invariably occurs in a conserved cluster of soxgenes ( soxJXYZAKBW; see below) that notably does not include an $f c c A$ /soxE homolog. Monomeric SoxJ/CT1015 protein is readily isolated from Cba. tepidum and has been shown to enhance thiosulfate oxidation in vitro by a SOX enzyme system reconstituted from SoxAXK, SoxYZ, and SoxB isolated from Cba. tepidum (Ogawa et al., 2010). SoxF isolated from P. pantotrophus also stimulates thiosulfate oxidation by a reconstituted enzyme system of Sox proteins isolated from P. pantotrophus (Friedrich et al., 2008). Thus, based on genetic and biochemical evidence, SoxJ in GSB appears to enhance thiosulfate oxidation by the SOX system. FccB and SoxJ from GSB have high sequence similarity and do not form obvious, monophyletic groups in a phylogenetic analysis (Figure 4A); therefore, they are distinguished primarily on the basis of genetic context (see above). However, when combined, all FccB and SoxJ sequences from GSB form a monophyletic group (Figure 4A). This suggests that the horizontal transfer of an ancestral gene into the GSB lineage (probably as $f c c A B$ ) only happened once and that SoxJ arose by duplication of FccB after this transfer to the GSB.

\section{SOX ENZYME SYSTEM}

Orthologs of some genes that encode the well-characterized, thiosulfate-oxidizing Sox enzyme system in P. pantotrophus (soxXYZABCDEFGH; Friedrich et al., 2005, 2008) were readily identified in seven of the GSB genomes (Table 1). All GSB capable of growth on thiosulfate contain soxgenes. However, in agreement with previous observations (Eisen et al., 2002; Frigaard and Bryant, 2008), the soxCD genes that encode the essential SoxCD component of the reaction mechanism in $P$. pantotrophus were absent from all GSB genomes. The soxgenes in GSB consistently occurred in a conserved cluster of eight genes, which were identical to the clusters originally identified in Cba. thiosulfatiphilum DSM 249 (Verté et al., 2002) and Cba. tepidum (Eisen et al., 2002). Because of their presence in this conserved cluster, two of the genes have been renamed soxJ(CT1015 in Cba. tepidum TLS; referred to as soxF2 in Cba. thiosulfatiphilum DSM 249 by Verté et al., 2002) and soxK (CT1020 in Cba. tepidum TLS; referred to as orf6 in Cba. thiosulfatiphilum DSM 249 by Verté et al., 2002). SoxJ is a flavoprotein that is homologous to FccB/SoxF (described above). SoxK is a component of the trimeric SoxAXK complex isolated from Cba. tepidum and is essential for efficient thiosulfate oxidation when using Sox enzymes isolated from $\mathrm{Cba}$. tepidum that are reconstituted in vitro (Ogawa et al., 2008). The presence of sox $\mathrm{K}$ in various bacteria is correlated with the occurrence of a SoxAX complex that comprises a monoheme SoxA and a monoheme SoxX; P. pantotrophus and many other bacteria have a diheme SoxA (Ogawa et al., 2008).

Among all sox-containing bacteria for which genome sequence data is currently available, the gene organization of the sox cluster

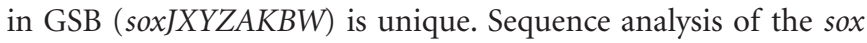

region in GSB suggested that there is only one promoter and that this promoter is located upstream of the soxJ gene (Figure 5). This observation suggests that the soxJXYZAKBW cluster probably is transcribed as a single mRNA. Consistent with this interpretation, transcription profiling shows that mRNA levels for all of the genes of this cluster are about 10-fold more abundant in cells growing on thiosulfate than on sulfide.

\section{THIOSULFATE UTILIZATION MAY BE ENCODED BY A MOBILE GENETIC ELEMENT}

A phylogenetic tree of concatenated Sox proteins from GSB is shown in Figure 4B. The eight proteins encoded by the soxJXYZAKBW cluster in GSB had largely congruent phylogeny (data not shown), which suggests that the cluster has been inherited intact in all GSB strains. The phylogeny of the Sox proteins within the Chlorobium and Chlorobaculum lineages (Figure 4B) was congruent with the cellular core phylogeny (Figure 2) with one exception: the Chl. phaeovibrioides DSM 265 Sox proteins grouped with the Sox proteins of the Chlorobaculum lineage (Figure 4B). This suggests that the sox cluster was horizontally transferred from a Chlorobaculum strain to an ancestor of Chl. phaeovibrioides DSM 265. It is also evident that the sox promoter region in Chl. phaeovibrioides DSM 265 is more similar to the sox promoter region in Cba. parvum DSM 263 than to the promoters in other GSB strains (Figure 5).

Figure 6 shows a comparison of syntenic regions of the genome in Chl. phaeovibrioides DSM 265 (sox ${ }^{+}$) and the very closely related Chl. luteolum DSM 273 ( $s o x^{-}$). A global comparison of these two strains suggested that these were the two most similar GSB among the 15 sequenced strains (Bryant et al., 2011). Figure 6 reveals that the sox genes in Chl. phaeovibrioides DSM 265 reside on an 11-kb island that is terminated by imperfect repeats (22-23 bp in length). Moreover, this island occurs in a region of the genome not otherwise related to sulfur metabolism. This 11-kb "sox island" harbors genes encoding a transposase, an integrase, and an RNA-directed DNA polymerase (reverse transcriptase), all of which are indicative of a mobile genetic element. The terminal imperfect inverted repeats suggest that the island was mobilized by the transposase. Integrase and reverse transcriptase usually do not occur on the same mobile element but the observation that the genes are located on a fragment flanked by inverted repeats suggests that they have the same origin. Reverse transcriptase occurs in a variety of mobile elements including retroviruses and bacterial retrons, suggesting that the mobile element could have been propagated via an RNA stage. No systems of this type, nor RNA or DNA viruses, have yet been characterized in GSB.

A 15-kb plasmid (accession number NC_002095) isolated from Cba. thiosulfatiphilum DSM 249 was reported to encode thiosulfate utilization when transformed into Chl. limicola DSM 245 (MéndezAlvarez et al., 1994). However, this plasmid does not contain sox genes and it is not clear how this plasmid could encode thiosulfate utilization (Frigaard and Bryant, 2008).

\section{DSR ENZYME SYSTEM}

The dissimilatory sulfite reductase (DSR) system is essential for oxidation of sulfur globules in Cba. tepidum (Holkenbrink et al., 2011) and Alc. vinosum (Dahl et al., 2005). Most GSB strains contain $d s r$ genes that occur as a single conserved gene cluster 


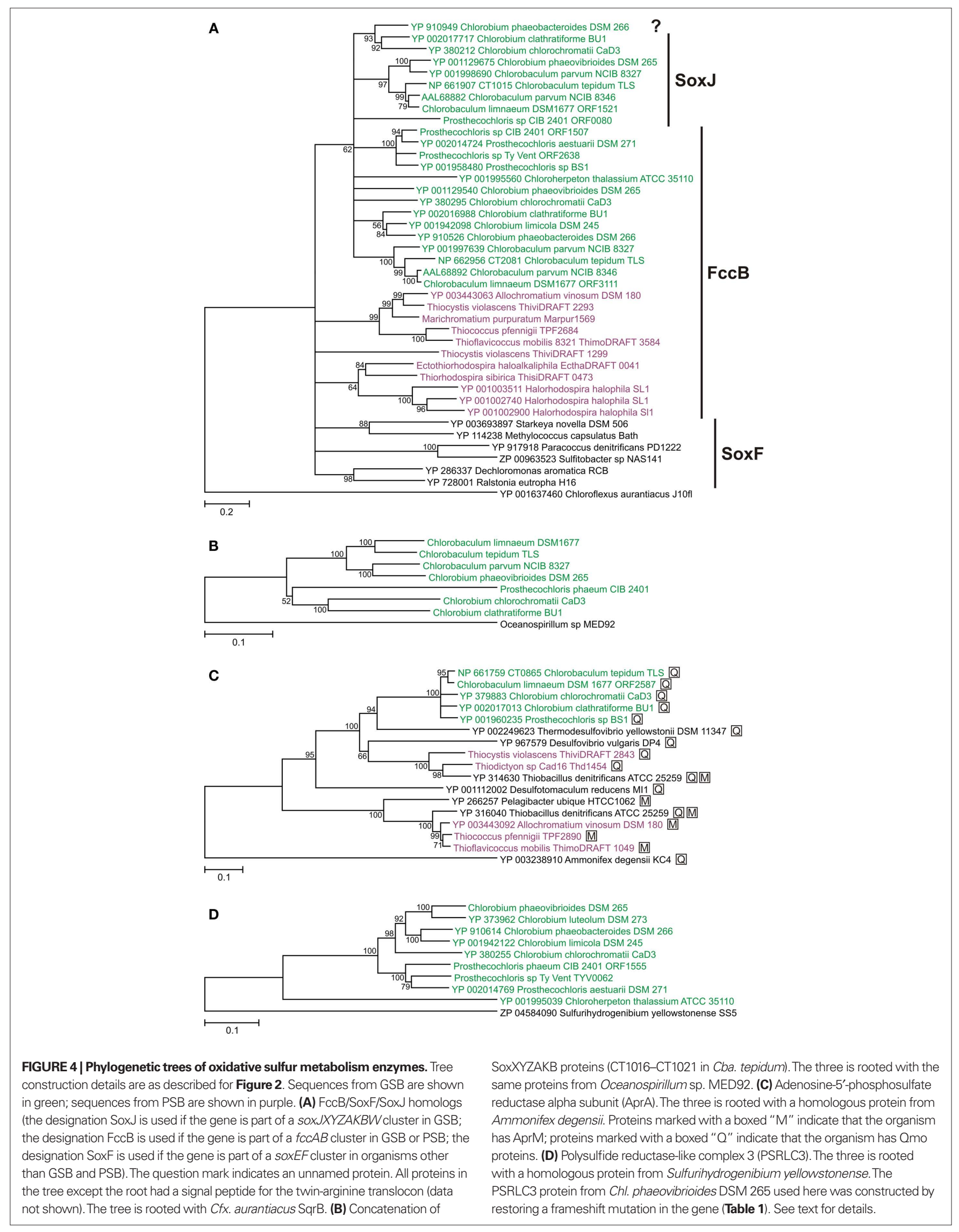


dsrNCABLUEFHTMKJOPVW (Table 1). One exception is Chl. ferrooxidans DSM 13031, which lacks all $d s r$ genes and most other genes related to oxidative sulfur metabolism (Table 1). This is consistent with the observation that this organism does not grow on reduced sulfur compounds (Heising et al., 1999). The only other strain that lacks all dsr genes is Chp. thalassium ATCC 35110, which has been reported to "slowly" consume sulfur globules (Gibson et al., 1984; see Discussion for further details). Cba. parvum DSM 263 has a single cluster of $d s r$ genes ( $d s r N C A B L U T M K J O P V W$ ), which lacks the $d s r E F H$ genes. This strain accumulates sulfur globules and does not produce sulfate when grown on sulfide (Kelly, 2008), which strongly suggests that the $d s r E F H$ gene products are essential for a functional DSR system.

With respect to gene organization, the $d s r$ gene cluster in $C b a$. tepidum is split and the $d s r C A B L$ genes are duplicated: $d s r N C A B$ LUTMKJOPVW (CT2251-CT2238) and dsrCABLEFH (CT0851CT0857; Eisen et al., 2002). Although the current draft genome sequence of Cba. limnaeum DSM 1677 does not allow a definitive view of the gene organization, it appears that this organism also has all $d s r$ genes, but that the $d s r$ genes are split and duplicated in the same manner as in Cba. tepidum. Interestingly, the $d s r$ gene

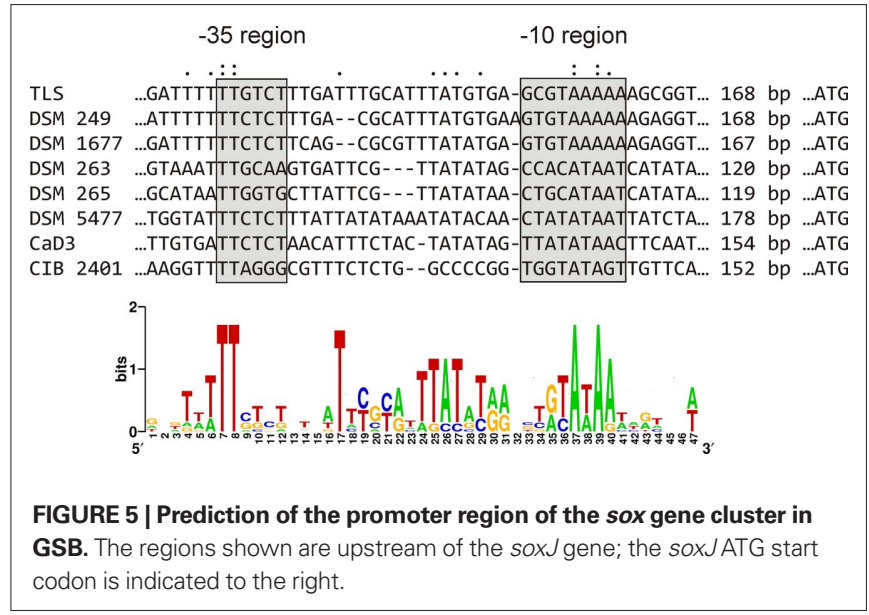

cluster in Cba. parvum DSM 263 is identical to the longest cluster in Cba. tepidum ( $d s r N C A B L U T M K J O P V W$ ). Thus, the clustering of $d s r$ genes is irregular in all investigated Chlorobaculum strains. One possible evolutionary scenario is that the canonical GSB $d s r$ cluster (dsrNCABLUEFHTMKJOPVW) split into two clusters ( $d s r N C A B L U T M K J O P V W$ and $d s r C A B L E F H)$ in a common ancestor of the investigated Chlorobaculum strains and that $\mathrm{Cba}$. parvum DSM 263 subsequently lost the short cluster and thereby the ability to oxidize sulfur globules. Cba. parvum DSM 263 is naturally transformable and apparently has an active homologous recombination system that may have facilitated this loss (Ormerod, 1988).

\section{ARE SOXCD AND DSR MUTUALLY EXCLUSIVE?}

The SOX system in GSB only partially oxidizes thiosulfate (Figure 1), whereas the SOX system in P. pantotrophus and many other bacteria completely oxidizes thiosulfate to sulfate (Friedrich et al., 2008). This difference is dependent on the presence of SoxCD in P. pantotrophus, which is absent from GSB. Complete oxidation of sulfur compounds to sulfate in GSB requires the DSR system (Figure 1; Holkenbrink et al., 2011).

We surveyed 1429 publicly available genome sequences ${ }^{14}$ for sox genes using BLASTP and known Sox protein sequences as queries. The retrieved sequences were then evaluated by sequence comparison, phylogenetic analysis, and gene clustering analyses to identify bona fide Sox proteins. We used the criterion that all Sox proteins of a given function must form a monophyletic clade, for which the clade was defined by a small number of query sequences.

The genomes were surveyed for SoxA/SoxB using BLASTP and the following query sequences (Meyer et al., 2007; Friedrich et al., 2008): YP_917913/YP_917914 (Paracoccus denitrificans PD1222), YP_144681/YP_144683 (Thermus thermophilus HB8), YP_487970/YP_487967 (Rhodopseudomonas palustris HaA2), YP_727992/YP_727989 (Ralstonia eutropha H16), NP_767651 (SoxA; Bradyrhizobium japonicum USDA 110), and NP_214237 (SoxB; Aquifex aeolicus VF5). The outgroup in phylogenetic

${ }^{14}$ http://www.ncbi.nlm.nih.gov/sutils/genom_table.cgi

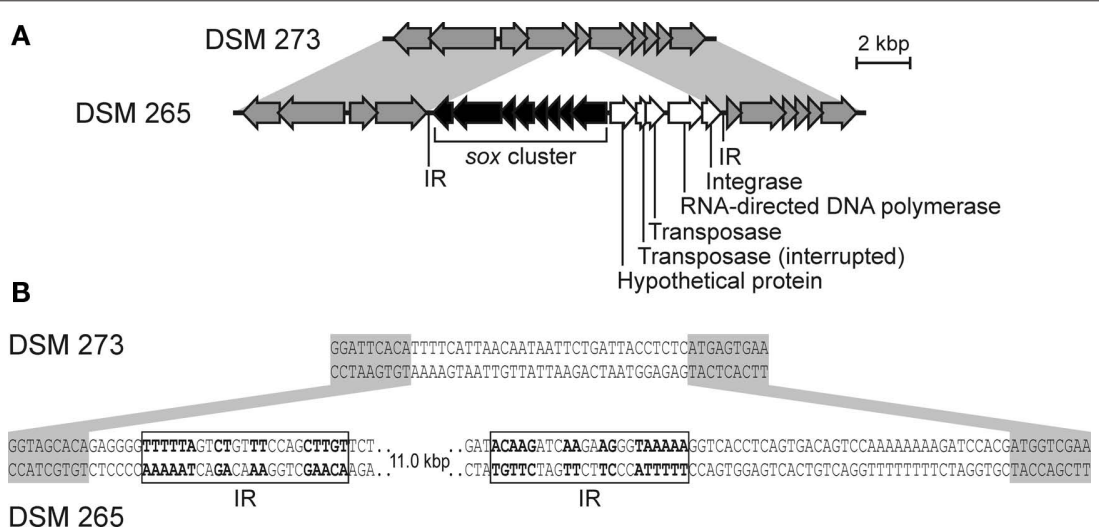

FIGURE 6 |The sox gene island in Chl. phaeovibrioides DSM 265. (A) Alignment of syntenic regions in Chl. luteolum DSM 273 and Chl. phaeovibrioides DSM 265. (B) Details of the sox gene island terminal sequences and insertion site. Genes marked in gray are not related to sulfur metabolism. Shades of gray indicate sequence similarity. IR, inverted repeat. 
analyses of SoxA and SoxB was ZP_03609782 (Campylobacter rectus RM3267) and ZP_02950408 (Clostridium butyricum 5521), respectively. In total, 127 genomes were identified that contained both soxA and $\operatorname{soxB}$ (56 Alphaproteobacteria, 26 Betaproteobacteria, 15 Gammaproteobacteria, 8 Epsilonproteobacteria, 8 Aquificales, 5 Chlorobiaceae, 5 Thermaceae, 3 Deltaproteobacteria, and 1 Magnetococcussp.). (Magnetococcus is member of the Proteobacteria whose subdivision affiliation is not resolved.) These genomes were then surveyed for SoxC/SoxD using BLASTP and the following query sequences (Friedrich et al., 2008): YP_917915/ YP_917916 (Paracoccus denitrificans PD1222), YP_144677/ YP_144676 (Thermus thermophilus HB8), YP_487966/YP_487965 (Rhodopseudomonas palustris HaA2), YP_727998/YP_841734/ YP_727997/YP_841733(Ralstonia eutrophaH16), and NP_770156/ NP_772761/NP_770157/NP_772760 (Bradyrhizobium japonicum USDA 110). Sequences that were more related to the homologous SorAB sulfite:cytochrome $c$ oxidoreductase were eliminated. SorA/ SorB queries used: AAF64400/AAF64401 (Starkeya novella DSM 506; Kappler et al., 2000) and YP_003892083/YP_003892084 (Sulfurimonas autotrophica DSM 16294). We found that most genomes (about 100 of the 127 genomes investigated) encoded SoxC and SoxD.

The DSR enzyme subunits DsrA and DsrB that function in sulfur oxidation (oxDsrAB) can be identified by sequence analysis, because these sequences form a monophyletic clade, which is clearly separated from the group of paralogous enzymes that function in sulfate reduction (redDsrAB; Loy et al., 2009). The 127 genomes that encoded SoxA and SoxB were surveyed for DsrA and DsrB using BLASTP and known DsrA and DsrB sequences as queries (Loy et al., 2009). The oxDsrA/oxDsrB queries used were NP_661746/NP_661747 (Cba.tepidum TLS), YP_003443222/ YP_003443223 (Alc. vinosum DSM 180), and YP_866063/ YP_866064 (Magnetococcus sp. MC-1). The redDsrA/redDsrB queries used were YP_967975/YP_967974 (Desulfovibrio vulgaris DP4), YP_001114514/YP_001114513 (Desulfotomaculum reducens MI-1), and NP_069259/NP_069260 (Archaeoglobus fulgidus DSM 4304). The obtained DsrA and DsrB sequences were easily identified and classified by sequence comparison and phylogenetic analysis. None of the 127 genomes that encoded SoxA and SoxB also encoded redDsrAB, which indicated that no sulfatereducing bacterium has the $\mathrm{SOX}$ system. In contrast, oxDsrAB genes were present in 15 of the genomes that encoded SoxA and SoxB. None of these 15 genomes also encoded soxCD. Thus, this survey showed that SoxCD and DSR are mutually exclusive. Specifically, these 15 organisms were: five strains of GSB (Table 1), Allochromatium vinosum DSM 180, Halorhodospira halophila SL1, Thioalkalivibrio sp. HL-EbGR7, Sideroxydans lithotrophicus ES-1, Thiobacillus denitrificans ATCC 25259, Beggiatoa sp. PS, Candidatus Vesicomyosocius okutanii HA, Candidatus Ruthia magnifica str. Cm, Magnetospirillum magnetotacticum MS-1, and Magnetococcus sp. MC-1.

Interestingly, some SoxAB-encoding genomes encode neither SoxCD nor DsrAB (e.g., all of the eight strains of Aquificales examined including Aquifex aeolicus VF5 (Ghosh et al., 2009), Hyphomonas neptunium ATCC 15444, Cupriavidus metallidurans $\mathrm{CH} 34$, and Ralstonia pickettii 12D). These organisms must use an unknown mechanism to regenerate the SOX system.

\section{OXIDATION OF SULFITE}

Although GSB cannot grow on sulfite (Frigaard and Dahl, 2009), the DSR system presumably produces sulfite, which must be oxidized (Figure 1). None of the 16 genomes listed in Table 1 encode homologs of the SorAB sulfite dehydrogenase (AAF64400/ AAF64401) known from Starkeya novella (Kappler et al., 2000).

One-third of the analyzed GSB strains encode adenosine-5'phosphosulfate (APS) reductase (APR; Table 1; Figure 4C; AprAB/ CT0865-CT0864 in Cba. tepidum TLS). This APR probably functions in sulfite oxidation and may be coupled with ATP sulfurylase (Sat) and the membrane-bound QmoABC oxidoreductase as depicted in Figure 1 (Frigaard and Dahl, 2009). These enzymes are encoded by a sat-aprBA-qmoABCgene cluster that is conserved in all GSB that have these genes (Table 1). None of the GSB investigated encodes the putative membrane-bound oxidoreductase AprM found in Alc. vinosum DSM 180 (Hipp et al., 1997). Deletion of the entire sat-aprBA-qmoABC gene cluster in Cba. tepidum TLS does not prevent the mutant strain from growth on sulfide and from producing sulfate (Holkenbrink, 2010). This observation suggests that even if APR functions in sulfite oxidation in GSB, it is not the only mechanism that oxidizes sulfite in Cba. tepidum TLS. One such possible mechanism is that sulfite reacts abiotically with oligosulfides to form thiosulfate, which subsequently is oxidized by the SOX system (Holkenbrink et al., 2011).

Polysulfide reductase-like complex 3 (PSRLC3) is a putative sulfite dehydrogenase that is found in some GSB (YP_910613 and YP_910614 in Chl. phaeobacteroides DSM 266; Frigaard and Bryant, 2008). In all Chlorobium strains (expect Chl. limicola DSM 245) the genes encoding PSRLC3 are located immediately upstream of the $d s r$ gene cluster (Frigaard and Dahl, 2009; data not shown), consistent with a functional link between PSRLC3 and oxidative sulfur metabolism. Sequence analysis showed that all sulfur-oxidizing GSB have either APR or PSRLC3, except Chl. chlorochromatii CaD3 that has both enzymes (Table 1). Analysis of the PsrA-like subunit of PSRLC3 showed that the phylogeny (Figure 4D) is congruent, at least at the genus level, with that of cellular core genes (Figure 2). This suggests that PSRLC3 was present in the last common ancestor of the investigated GSB.

Like the GSB, PSB apparently do not use a single mechanism to oxidize sulfite. Alc. vinosum DSM 180 has APR that apparently is coupled with the putative membrane-bound oxidoreductase AprM (Hipp et al., 1997). Among the PSB examined here, three strains encode AprABM (Alc. vinosum DSM 180, Tco. pfennigii 4520, Tfc. mobilis 8321) and two strains encode AprAB-QmoAB (Thiodictyon sp. Cad16, Tcs. violascens DSM 198; Figure 4C). Sat, AprAB, and QmoAB were not found in any of the Ectothiorhodospiraceae (Ect. haloalkaliphila ATCC 51935, Hlr. halophila SL1, Trs. sibirica ATCC 700588) or Mch. purpuratum DSM 1591. SorAB and PSRLC3 have not yet been found in any PSB strain.

\section{DISCUSSION}

The congruent phylogenies of rRNA sequences and conserved housekeeping functions provide a solid foundation for understanding the evolution of the GSB lineage (Figures 2A,B). A selection of genes encoding physiological traits characteristic of GSB had overall congruent phylogenies with the rRNA and house-keeping functions, at least to the genus level (Figure 2C; and examples mentioned in Section "Results"). This suggests that these physiological functions were present in the last common ancestor of known extant GSB and 
were passed on only by vertical descent. This includes elements of phototrophy, carbon fixation, nitrogen fixation, and hydrogen uptake (although a few strains have apparently lost their hup genes). Most of the genes encoding components of oxidative sulfur metabolism in GSB, on the other hand, have very different evolutionary histories.

The DSR system is not present in the earliest diverging GSB (Chp. thalassium) and appears to have been acquired by horizontal gene transfer, in part from other sulfide-oxidizing organisms and in part from sulfate-reducing organisms, after divergence of Chloroherpeton (Sander et al., 2006). It is not clear from the literature how, or whether, sulfide is oxidized to sulfate in Chp. thalassium (Gibson et al., 1984). The question arises because the DSR system is essential for this process in other GSB (Holkenbrink et al., 2011). It is also not clear how the sulfite produced by the DSR system is oxidized. A single sulfite oxidation mechanism present in all thiotrophic GSB or PSB has not been identified. Phylogenetic analyses suggest that the putative sulfite dehydrogenase PSRLC3 was present in the last common ancestor of the investigated GSB (Figure 4D). It is an attractive hypothesis that the APR system replaced the PSRLC3 in sulfite oxidation by horizontal gene transfer in a few GSB strains and that this acquisition was selected for due to increased energy conservation in the APR pathway as compared to the PSRLC3 pathway (Figure 1). A genetic transfer mechanism of the sat-apr-qmo genes has not been identified but a similar cluster of genes with high sequence similarity occurs in sulfate-reducing bacteria that typically co-occur with GSB in favorable habitats (Meyer and Kuever, 2007).

The SOX system for thiosulfate oxidation is widespread among very divergent organisms (Meyer et al., 2007). Formation of thiosulfate in the environment occurs under various conditions, such as by mixing of sulfide and $\mathrm{O}_{2}$ (abiotic formation) and as a byproduct of sulfide oxidation in GSB and PSB (Holkenbrink et al., 2011). The SOX system is present only in some GSB strains and appears to have been acquired by horizontal gene transfer from members of the Proteobacteria (Meyer et al., 2007), and subsequently exchanged horizontally within the GSB lineage (Figure 4B). The SOX system in most organisms other than GSB and PSB includes the SoxCD complex, which together with other Sox components allows complete oxidation of one molecule of thiosulfate to two molecules of sulfate in the periplasm while producing reduced cytochrome $c$. However, SoxCD is not part of the SOX system in GSB and PSB, in which the DSR system completes the oxidation of the sulfane moiety of thiosulfate. The DSR-dependent mechanism presumably conserves more of the chemical energy available to the organism, because the reducing equivalents from the sulfane moiety are recovered as reduced isoprenoid quinones in the cytoplasmic membrane. In addition, chemical energy may be conserved by substrate-level phosphorylation by the APR system (Figure 1). If DSRdependent thiosulfate oxidation indeed conserves more energy than SoxCD-dependent thiosulfate oxidation, this may explain why loss of soxCD genes has occurred in bacteria that contain both the SOX and DSR systems. Our genome sequence analyses showed that loss of the soxCD genes has occurred independently in unrelated DSRcontaining organisms with divergent Sox systems, including GSB, PSB, the betaproteobacterium Thiobacillus denitrificans ATCC 25259, and the divergent proteobacterium Magnetococcus sp. MC-1.

The current collection of cultured GSB strains exhibits very limited physiological variation (Imhoff, 2003). Limited success and approaches to isolation of axenic cultures could explain part of this observation. However, many cases have been described where the GSB that are found in environmental studies of lakes and sediments (where one expects to find GSB), are similar to cultured strains (at least at the level of SSU rRNA sequence similarity, e.g., Koizumi et al., 2004; Manske et al., 2005; Alexander and Imhoff, 2006; Gregersen et al., 2009). Therefore, it is likely that these strains contain the DSR system. The DSR system provides GSB with an improved utilization of reduced sulfur compounds that is likely to be advantageous in sulfide-limited and energy-limited environments (Holkenbrink et al., 2011). Although the DSR system appears to allow GSB to prosper in certain environments, the DSR system may not be important to GSBlike organisms in environments with high sulfide concentrations or to organisms that participate in symbioses with microbes that reduce elemental sulfur. The former reason may in part explain why $\mathrm{Cba}$. parvum has lost three essential genes $(d s r E F H)$ and can no longer oxidize elemental sulfur (Table 1). GSB that can grow by oxidation of $\mathrm{Fe}^{2+}$ or $\mathrm{H}_{2}$ as the sole electron donor also do not need DSR (Heising et al., 1999). Chp. thalassium is another GSB that thrives without DSR, although the ecophysiology of this organism is not yet described in detail (Gibson et al., 1984). It would be very interesting to know (i) if there are phototrophic relatives of GSB without DSR that are metabolically important community members; (ii) under which environmental conditions such GSB thrive; and (iii) the detailed metabolism and ecophysiology of such GSB. Cultivation-independent approaches (including metagenomics) and novel approaches to isolation of pure cultures may provide information on these issues.

\section{CONCLUSION}

The phylogenetic analyses presented here and elsewhere (Sander et al., 2006; Meyer and Kuever, 2007; Meyer et al., 2007; Frigaard and Bryant, 2008; Frigaard and Dahl, 2009; Loy et al.,2009) strongly suggest that the currently known and characterized GSB have obtained many of their oxidative sulfur metabolism genes by horizontal gene transfer from a variety of chemotrophic bacteria that either oxidize sulfur compounds or reduce sulfur compounds. In addition, certain steps in the oxidative sulfur metabolism in some GSB appear to have been replaced with pathways that we hypothesize improve conservation of chemical energy by introducing substrate-level phosphorylation reactions and increased transmembrane proton transfer (e.g., DSR replacing SoxCD in thiosulfate oxidation and APR putatively replacing PSRLC3 in sulfite oxidation). Although progress has been made in understanding the enzymes and pathways involved in oxidative sulfur metabolism in GSB, many essential aspects are still not understood. This includes the mechanisms for formation and consumption of sulfur globules, sulfite oxidation, and SQR-independent sulfide consumption. The rapidly increasing amount of genome sequence information for both GSB and PSB will surely improve our understanding of these processes.

\section{ACKNOWLEDGMENTS}

Niels-Ulrik Frigaard was supported by a grant from the Danish Natural Science Research Council (21-04-0463). Donald A. Bryant was supported by grants from the U.S. National Science Foundation (MCB-0523100), U.S. Department of Energy (DE-FG0294ER20137) and NASA Exobiology (NNX09AM87G). Donald A. Bryant and Niels-Ulrik Frigaard thank the Joint Genome Institute for their assistance in generating draft genomes of some of the GSB and PSB discussed here. 


\section{REFERENCES}

Alexander, B., Andersen, J. H., Cox, R. P., and Imhoff, J. F. (2002). Phylogeny of green sulfur bacteria on the basis of gene sequences of 16S rRNA and of the Fenna-Matthews-Olson protein. Arch. Microbiol. 178, 131-140.

Alexander, B., and Imhoff, J. F. (2006). Communities of green sulfur bacteria in marine and saline habitats analyzed by gene sequences of $16 \mathrm{~S}$ rRNA and Fenna-Matthews-Olson protein. Int. Microbiol. 9, 259-266.

Azai, C., Tsukatani, Y., Harada, J., and Oh-Oka, H. (2009). Sulfur oxidation in mutants of the photosynthetic green sulfur bacterium Chlorobium tepidum devoid of cytochrome c-554 and SoxB. Photosyn. Res. 100, 57-65.

Bagos, P. G., Nikolaou, E. P., Liakopoulos, T. D., and Tsirigos, K. D. (2010). Combined prediction of Tat and Sec signal peptides with hidden Markov models. Bioinformatics 26, 2811-2817.

Brito, J. A., Sousa, F. L., Stelter, M., Bandeiras, T.M., Vonrhein, C., Teixeira, M., Pereira, M. M., and Archer, M. (2009). Structural and functional insights into sulfide:quinone oxidoreductase. Biochemistry 48, 5613-5622.

Bronstein, M., Schütz, M., Hauska, G., Padan, E., and Shahak, Y. (2000). Cyanobacterial sulfide-quinone reductase: cloning and heterologous expression. J. Bacteriol. 182, 3336-3344.

Brune, D. C. (1989). Sulfur oxidation by phototrophic bacteria. Biochim. Biophys. Acta 975, 189-221.

Bryant, D. A., Klatt, C. G., Frigaard, N.-U., Liu, Z., Li, T., Zhao, F., Garcia Costas, A. M., Overmann, J., and Ward, D. M. (2011). "Comparative and functional genomics of anoxygenic green bacteria from the taxa Chlorobi, Chloroflexi, and Acidobacteria," in Advances in Photosynthesis and Respiration, Vol. 33, Functional Genomics and Evolution of Photosynthetic Systems, eds R. L. Burnap and W. Vermaas (Dordrecht: Springer), in press.

Bryantseva, I. A., Gorlenko, V. M., Kompantseva, E. I., Imhoff, J. F., Süling, J., and Mityushina, L. (1999). Thiorhodospira sibirica gen. nov., sp. nov., a new alkaliphilic purple sulfur bacterium from a Siberian Soda lake. Int. J. Syst. Bacteriol. 49, 697-703.

Carver, T., Berriman, M., Tivey, A., Patel, C., Böhme, U., Barrell, B. G., Parkhill, J., and Rajandream, M. A. (2008). Artemis and ACT: viewing, annotating and comparing sequences stored in a relational database. Bioinformatics 24, 2672-2676.

Chan, L.-K., Morgan-Kiss, R., and Hanson, T. E. (2008). "Genetic and proteomic studies of sulfur oxidation in Chlorobium tepidum (syn.
Chlorobaculum tepidum)," in Sulfur Metabolism in Phototrophic Organisms, R. Hell, C. Dahl, D. Knaff, and T. Leustek (New York: Springer), 363-379.

Chan, L. K., Morgan-Kiss, R. M., and Hanson, T.E. (2009). Functional analysis of three sulfide:quinone oxidoreductase homologs in Chlorobaculum tepidum. J. Bacteriol. 191, 1026-1034.

Crooks, G. E., Hon, G., Chandonia, J. M., and Brenner, S. E. (2004). WebLogo: a sequence logo generator. Genome Res. 14, 1188-1190.

Dahl, C., Engels, S., Pott-Sperling, A. S., Schulte, A., Sander, J., Lübbe, Y., Deuster, O., and Brune, D. C. (2005). Novel genes of the $d s r$ gene cluster and evidence for close interaction of Dsr proteins during sulfur oxidation in the phototrophic sulfur bacterium Allochromatium vinosum. J. Bacteriol. 187, 1392-1404.

Demerec, M., Adelberg, E. A., Clark, A. J., and Hartman, P.E. (1966). A proposal for a uniform nomenclature in bacterial genetics. Genetics 54, 61-76.

Efremov, R. G., Baradaran, R., and Sazanov, L. A. (2010). The architecture of respiratory complex I. Nature 465, 441-445.

Eisen, J. A., Nelson, K. E., Paulsen, I. T., Heidelberg, J. F., Wu, M., Dodson, R. J., Deboy, R., Gwinn, M. L., Nelson, W. C., Haft, D. H., Hickey, E. K., Peterson, J. D., Durkin, A.S., Kolonay, J. L., Yang, F., Holt, I., Umayam, L. A., Mason, T., Brenner, M., Shea, T. P., Parksey, D., Nierman, W. C., Feldblyum, T. V., Hansen, C. L., Craven, M. B., Radune, D., Vamathevan, J., Khouri, H., White, O., Gruber, T. M., Ketchum, K. A., Venter, J. C., Tettelin, H., Bryant, D. A., and Fraser, C.M. (2002). The complete genome sequence of Chlorobium tepidum TLS, a photosynthetic, anaerobic, green-sulfur bacterium. Proc. Natl. Acad. Sci. U.S.A. 99, 9509-9514.

Emanuelsson, O., Brunak, S., von Heijne, G., and Nielsen, H. (2007). Locating proteins in the cell using TargetP, SignalP and related tools. Nat. Protoc. 2, 953-971.

Friedrich, C. G., Bardischewsky, F., Rother, D., Quentmeier, A., and Fischer, J. (2005). Prokaryotic sulfur oxidation. Curr. Opin. Microbiol. 8, 253-259.

Friedrich, C. G., Quentmeier, A., Bardischewsky, F., Rother, D., Orawski, G., Hellwig, P., and Fischer, J. (2008). "Redox control of chemotrophic sulfur oxidation in Paracoccus pantotrophus," in Microbial Sulfur Metabolism, eds C. Dahl and C. G. Friedrich, (Heidelberg: Springer),139-150.

Frigaard, N.-U., and Bryant, D. A. (2008). "Genomics insights into the sulfur metabolism of phototrophic green sulfur bacteria," in Advances in Photosynthesis and Respiration, Vol.
27, eds R. Hell, C. Dahl, D. B. Knaff, and T. Leustek (Heidelberg: Springer), 337-355.

Frigaard, N.-U., and Dahl, C. (2009). "Sulfur metabolism in phototrophic sulfur bacteria," in Advances in Microbial Physiology, ed. R. K. Poole (London: Academic Press), 103-200.

Frigaard, N.-U., Gomez Maqueo Chew,A. Li, H., Maresca, J. A., and Bryant, D. A (2003). Chlorobium tepidum: insights into the structure, physiology, and metabolism of a green sulfur bacterium derived from the complete genome sequence. Photosyn. Res. 78, 93-117.

Garrity, G. M., and Holt, J. G. (2001). "Phylum BXI. Chlorobi phy. nov.," in Bergey's Manual of Systematic Bacteriology, 2nd Edn., Vol. 1, eds D. R. Boone and R. W. Castenholz (New York, NY: Springer-Verlag), 601-623.

Ghosh, W., Mallick, S., and DasGupta, S K. (2009). Origin of the Sox multienzyme complex system in ancient thermophilic bacteria and coevolution of its constituent proteins. Res. Microbiol. 160, 409-420.

Gibson, J., Pfennig, N., and Waterbury, J. B. (1984). Chloroherpeton thalassium gen. nov. et spec. nov., a nonfilamentous, flexing and gliding green sulfur bacterium. Arch. Microbiol. 138 96-101.

Gomez Maqueo Chew,A., Frigaard, N.-U., and Bryant, D. A. (2009). Mutational analysis of three $b c h H$ paralogs in (bacterio-)chlorophyll biosynthesis in Chlorobaculum tepidum. Photosyn. Res. 101, 21-34.

Gregersen, L.H., Habicht, K. S., Peduzzi, S. Tonolla, M., Canfield, D. E., Miller, M. Cox, R. P., and Frigaard, N.-U. (2009) Dominance of a clonal green sulfur bacterial population in a stratified lake. FEMS Microbiol. Ecol. 70, 30-41. Hanson, T. E., and Tabita, F. R. (2001) A ribulose-1,5-bisphosphate carboxylase/oxygenase (RubisCO)-like protein from Chlorobium tepidum that is involved with sulfur metabolism and the response to oxidative stress. Proc Natl. Acad. Sci. U.S.A. 98, 4397-4402.

Heising, S., Richter, L., Ludwig, W., and Schink, B. (1999). Chlorobium ferrooxidans sp. nov., a phototrophic green sulfur bacterium that oxidizes ferrous iron in coculture with a "Geospirillum" sp. strain. Arch Microbiol 172, 116-124.

Hipp, W. M., Pott, A. S., Thum-Schmitz N., Faath, I., Dahl, C., and Trüper, H.G. (1997). Towards the phylogeny of APS reductases and sirohaem sulfite reductases in sulfate-reducing and sulfuroxidizing prokaryotes. Microbiology 143, 2891-2902.

Holkenbrink, C. (2010). Thiotrophic sulfur metabolism in green sulfur bacteria. Master's thesis, University of Copenhagen, Copenhagen, Denmark.
Holkenbrink, C., Ocón Barbas, S., Mellerup, A., Otaki, H., and Frigaard, N.-U.(2011). Sulfur globule oxidation in green sulfur bacteria is dependent on the dissimilatory sulfite reductase system. Microbiology 157, 1229-1239.

Iino, T., Mori, K., Uchino, Y., Nakagawa, T., Harayama, S., and Suzuki, K. (2010). Ignavibacterium album gen. nov., sp. nov., a moderately thermophilic anaerobic bacterium isolated from microbial mats at a terrestrial hot spring and proposal of Ignavibacteria classis nov., for a novel lineage at the periphery of green sulfur bacteria. Int. J. Syst. Evol. Microbiol. 60, 1376-1382.

Imhoff, J. F. (2003). Phylogenetic taxonomy of the family Chlorobiaceae on the basis of 16S rRNA and fmo (Fenna-Matthews-Olson protein) gene sequences. Int. J. Syst. Evol. Microbiol. 53, 941-951.

Imhoff, J. F. (2008). "Systematics of anoxygenic phototrophic bacteria," in Sulfur Metabolism in Phototrophic Organisms, eds R. Hell, C. Dahl, D. Knaff, and T. Leustek (Dordrecht: Springer), 269-287.

Kappler, U., Bennett, B., Rethmeier, J., Schwarz, G., Deutzmann, R., McEwan, A. G., and Dahl, C. (2000). Sulfite:cytochrome $c$ oxidoreductase from Thiobacillus novellus - purification, characterization, and molecular biology of a heterodimeric member of the sulfite oxidase family. J. Biol. Chem. 275, 13202-13212.

Kelly, D. P. (2008). Stable sulfur isotope fractionation by the green bacterium Chlorobaculum parvum during photolithoautotrophic growth on sulfide. Pol. J. Microbiol. 57, 275-279.

Klatt, C. G., Wood, J. M., Rusch, D. B., Bateson, M. M., Heidelberg, J. F., Bryant, D. A., and Ward, D. W. (2011) Metagenomic analyses of phototrophic hot spring microbial mat communities. ISME J. (in press).

Koizumi, Y., Kojima, H., and Fukui, M. (2004). Dominant microbial composition and its vertical distribution in saline meromictic Lake Kaiike (Japan) as revealed by quantitative oligonucleotide probe membrane hybridization. Appl. Environ. Microbiol. 70, 4930-4940.

Kusai, K., and Yamanaka, T. (1973). The oxidation mechanisms of thiosulphate and sulphide in Chlorobium thiosulphatophilum: roles of cytochrome $c$-551 and cytochrome $c$-553. Biochim. Biophys. Acta 325, 304-314.

Liu, Z., Klatt, C. G., Wood, J., Rusch, D. B., Wittekindt, N., Tomsho, L., Schuster, S. C., Ward, D. M., and Bryant, D. A. (2011). Metatranscriptomic analyses of a hot spring microbial mat reveal temporal transcription differences 
among oxygenic and anoxygenic phototrophs. ISME J. (in press).

Loy,A., Duller, S., Baranyi, C., Mussmann, M., Ott, J., Sharon, I., Béjà, O., Le Paslier, D., Dahl, C., and Wagner, M. (2009). Reverse dissimilatory sulfite reductase as phylogenetic marker for a subgroup of sulfur-oxidizing prokaryotes. Environ. Microbiol. 11, 289-299.

Manske, A. K., Glaeser, J., Kuypers, M. A. M., and Overmann, J. (2005). Physiology and phylogeny of green sulfur bacteria forming a monospecific phototrophic assemblage at a depth of 100 meters in the Black Sea. Appl. Environ. Microbiol. 71, 8049-8060.

Marcia, M., Ermler, U., Peng, G., and Michel, H. (2009). The structure of Aquifex aeolicus sulfide:quinone oxidoreductase, a basis to understand sulfide detoxification and respiration. Proc. Natl. Acad. Sci. U.S.A. 106, 9625-9630.

Marcia, M., Ermler, U., Peng, G., and Michel, H. (2010). A new structurebased classification of sulfide:quinone oxidoreductases. Proteins 78, 1073-1083.

Méndez-Alvarez, S., Pavón, V., Esteve, I., Guerrero, R., and Gaju, N. (1994). Transformation of Chlorobium limicola by a plasmid that confers the ability to utilize thiosulfate. J. Bacteriol. 176, 7395-7397.

Meyer, B., Imhoff, J. F., and Kuever, J. (2007). Molecular analysis of the distribution and phylogeny of the sox $B$ gene among sulfur-oxidizing bacteria - evolution of the Sox sulfur oxidation enzyme system. Environ. Microbiol. 9, 2957-2977.
Meyer, B., and Kuever, J. (2007). Phylogeny of the alpha and beta subunits of the dissimilatory adenosine-5' phosphosulfate (APS) reductase from sulfate-reducing prokaryotes origin and evolution of the dissimilatory sulfate-reduction pathway. Microbiology 153, 2026-2044.

Nübel, T., Klughammer, C., Huber, R., Hauska, G., and Schütz, M. (2000). Sulfide:quinone oxidoreductase in membranes of the hyperthermophilic bacterium Aquifex aeolicus (VF5). Arch. Microbiol. 173, 233-244.

Ogawa, T., Furusawa, T., Nomura, R., Seo, D., Hosoya-Matsuda, N., Sakurai, H., and Inoue, K. (2008). SoxAX binding protein, a novel component of the thiosulfate oxidizing multienzyme system in the green sulfur bacterium Chlorobium tepidum. J. Bacteriol. 190, 6097-6110.

Ogawa, T., Furusawa, T., Shiga, M., Seo, D., Sakurai, H., and Inoue, K. (2010). Biochemical studies of a soxF-encoded monomeric flavoprotein purified from the green sulfur bacterium Chlorobaculum tepidum that stimulates in vitro thiosulfate oxidation. Biosci. Biotechnol. Biochem. 74, 771-780.

Ormerod, J. G. (1988). "Natural genetic transformation in Chlorobium," in Green Photosynthetic Bacteria, eds J. M. Olson, J. G. Ormerod, J. Amesz, E. Stackebrandt, and H. G. Trüper (New York: Plenum), 315-319.

Overmann, J. (2000). "The family Chlorobiaceae," in The Prokaryotes: An Evolving Electronic Resource for the Microbiological Community, 3rd Edn., release 3.1 (New York: SpringerVerlag). http://link.springer-ny.com/ link/service/books/10125/
Overmann, J. (2008). "Ecology of phototrophic sulfur bacteria," in Sulfur Metabolism in Phototrophic Organisms, eds R. Hell, C. Dahl, D. B. Knaff, and T. Leustek (Dordrecht: Springer) 375-396.

Reinartz, M., Tschäpe, J., Brüser, T. Trüper, H. G., and Dahl, C. (1998). Sulfide oxidation in the phototrophic sulfur bacterium Chromatium vinosum. Arch. Microbiol. 170, 59-68.

Sakurai, J., Ogawa, T., Shiga, M., and Inoue, K. (2010). Inorganic sulfur oxidizing system in green sulfur bacteria. Photosyn. Res. 104, 163-176.

Sander, J., Engels-Schwarzlose, S., and Dahl, C. (2006). Importance of the DsrMKJOP complex for sulfur oxidation in Allochromatium vinosum and phylogenetic analysis of related complexes in other prokaryotes. Arch. Microbiol. 186, 357-366.

Sazanov, L. A., and Hinchliffe, P. (2006) Structure of the hydrophilic domain of respiratory complex I from Thermus thermophilus. Science 311, 1430-1436.

Schütz, M., Maldener, I., Griesbeck, C., and Hauska, G. (1999). Sulfidequinone reductase from Rhodobacter capsulatus: requirement for growth, periplasmic localization, and extension of gene sequence analysis. $J$. Bacteriol. 181, 6516-6523.

Shahak, Y., and Hauska, G. (2008) "Sulfide oxidation from Cyanobacteria to humans: sulfide-quinone oxidoreductase (SQR)," in Sulfur Metabolism in Phototrophic Organisms, eds R. Hell, C. Dahl, D. B. Knaff, and T. Leustek(Dordrecht:Springer),319-335.

Tamura, K., Dudley, J., Nei, M., and Kumar, S. (2007). MEGA4: molecular evolutionary genetics analysis
(MEGA) software version 4.0. Mol. Biol. Evol. 24, 1596-1599.

Van de Weghe, J. G., and Ow, D. W. (1999). A fission yeast gene for mitochondrial sulfide oxidation. J. Biol. Chem. 274 13250-13257.

Verté, F., Kostanjevecki, V., De Smet, L., Meyer, T. E., Cusanovich, M. A., and Van Beeumen, J. J. (2002). Identification of a thiosulfate utilization gene cluster from the green phototrophic bacterium Chlorobium limicola. Biochemistry 41, 2932-2945.

Yagi, T., and Matsuno-Yagi, A. (2003). The proton-translocating $\mathrm{NADH}$-quinone oxidoreductase in the respiratory chain: the secret unlocked. Biochemistry 42, 2266-2274.

Conflict of Interest Statement: The authors declare that the research was conducted in the absence of any commercial or financial relationships that could be construed as a potential conflict of interest.

Received: 16 February 2011; accepted: 11 May 2011; published online: 24 May 2011. Citation: Gregersen LH, Bryant DA and Frigaard N-U (2011) Mechanisms and evolution of oxidative sulfur metabolism in green sulfur bacteria. Front. Microbio. 2:116. doi: 10.3389/fmicb.2011.00116 This article was submitted to Frontiers in Microbial Physiology and Metabolism, a specialty of Frontiers in Microbiology. Copyright (C) 2011 Gregersen, Bryant and Frigaard. This is an open-access article subject to a non-exclusive license between the authors and Frontiers Media SA, which permits use, distribution and reproduction in other forums, provided the original authors and source are credited and other Frontiers conditions are complied with. 